\title{
MID-IR EMISSION OF LOW-METALLICITY GALAXIES
}

\author{
S. PLANTE \\ Dept. de Physique, Université Laval \\ Cité Universitaire, Ste-Foy, $P Q$, Canada G1K $7 P 4$ \\ M. SAUVAGE \\ CEA $/ D S M / D A P N I A /$ Service d'Astrophysique \\ C.E. Saclay, 91191 Gif sur Yvette CEDEX, France
}

AND

D. KUNTH

Institut d'Astrophysique

98bis Bd Arago, 75014 Paris, France

\section{The influence of star formation in NGC 595}

NGC 595 is a giant HII region located in the western part of the spiral galaxy M 33. It is the second in importance in this galaxy, after NGC 604. At $0.84 \mathrm{Mpc}, H S T$ is able to resolve its stellar content. Malumuth et al. (1996) obtained HST UV, U, B and V images of this region and derived an ionizing luminosity of $5 \times 10^{50} \mathrm{phot} \mathrm{s}^{-1}$, and an average reddening $\mathrm{E}_{B-V}=0.36 \pm 0.28 \mathrm{mag}$. The stars are mostly concentrated in the central part of the region, where little emission of gas is seen (the ionized gas lies more in a shell around the stars, figure 1a). Wilson \& Scoville (1993) showed the molecular gas to be situated in the south-east part of the region, just outside of the bright knot of stars. Viallefond et al. (1986) found a reddening gradient in the north-east/south-west direction by observing the HI gas, which was confirmed by Malumuth $e t$ al. (1996) with stellar photometry.

We obtained ISO images for NGC 595 in the 5.0 to $8.5 \mu \mathrm{m}$ range. The emission in this spectral range is dominated by the so-called $P A H$ bands. Current interpretation of these has them originating from stochastically heated molecules. Two of these bands are located in the range observed, at $6.2 \mu \mathrm{m}$ and $7.7 \mu \mathrm{m}$. Stochastic heating implies that the in-band flux is directly proportional to the number of photons absorbed by the molecules. For typical HiI regions, Cohen et al. (1989) found 0.58 for the $I_{6.2} / I_{7.7}$ in-band ratio. However many processes, ionization, dehydrogenation, can modify this ratio. Furthermore, an underlying continuum is present though its exact origin is unknown.

\section{Data Reduction}

Three different filters have been used to cover this wavelength range: LW4 (5.5-6.5 $\mu \mathrm{m})$, LW5 (6.5$7.0 \mu \mathrm{m})$ and LW6 $(7.0-8.5 \mu \mathrm{m})$. The first one essentially contains the $6.2 \mu \mathrm{m}$ band, the second a pseudo-continuum in-between and the third, the $7.7 \mu \mathrm{m}$ band. We used CIA ${ }^{1}$ to reduce the data. Cosmic rays where removed with a multi-resolution based algorithm. We used an iterative technique to build a flat-field from the LW6 image, which had the best signal-to-noise ratio. We used this flat-field to correct our three images, as the wavelength range is small. Transient correction has been done with an inversion technique which takes into account the fact that we had different integration times for each of the filters. For details on ISOCAM data reduction, see Starck et al. (1998).

\footnotetext{
${ }^{1}$ CIA, CAM Interactive Analysis, is a joint development by the ESA Astrophysics Division and the ISOCAM consortium led by the ISOCAM P.I., C. Cesarsky, Direction des Sciences de la Matière, C.E.A., France.
} 

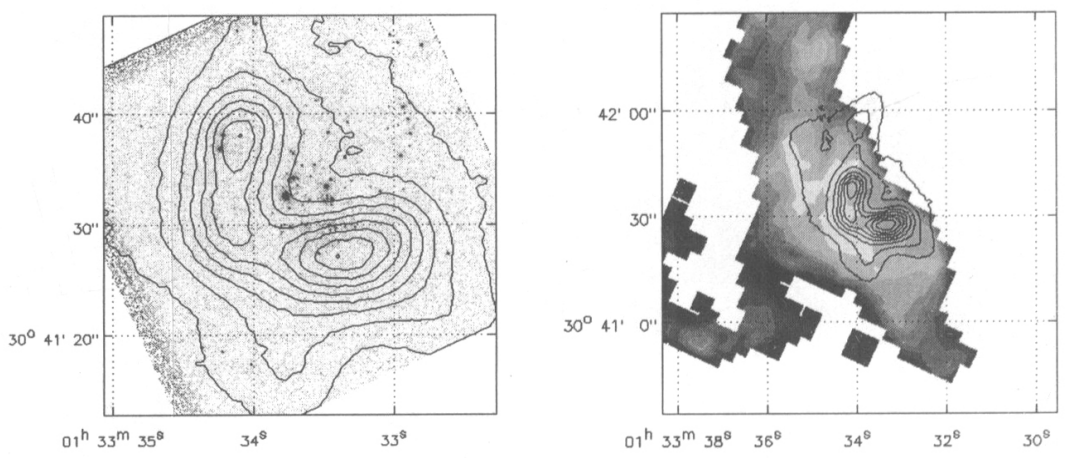

Figure 1. For both figures, North is on top and East is to the left, and the equinox is J2000.0. (a) - left: H $\alpha$ image on WFPC2 B-band image from Malumuth et al. (1996). Contours and grey levels are linearly spaced. (b)- right: In grey levels we represent the LW4/LW6 color ratio (logarithmic). For display reasons, the ISO maps have been resampled to $0.3^{\prime \prime}$ pixels but the real pixel size is $3^{\prime \prime}$. Overlaid on this map is the $\mathbf{H} \alpha$ image of $(\mathrm{a})$. Although the two distributions are related, the ISO emission is more extended and displaced in the south-east direction.

\section{Analysis}

Metcalfe et al. (1996) obtained an ISO spectrum of Haro 3, a Wolf-Rayet dwarf galaxy. Their spectrum shows clearly the two PAH bands. The spectrum of NGC 595 should in principle be similar, as both regions harbor the same energy sources (i.e. massive stars). The images we obtained show that the flux density in the LW5 filter (the pseudo continuum) is higher than that in the LW4 filter (the $6.2 \mu \mathrm{m}$ band) and almost as high as that in the LW6 filter (the $7.7 \mu \mathrm{m}$ band). We tried to reconstruct for each pixel the 5.0-8.5 $\mu \mathrm{m}$ spectrum of NGC 595 by fixing the FWHM of the two PAH bands ( $0.4 \mu \mathrm{m}$ for the $6.2 \mu \mathrm{m}$ band and $0.9 \mu \mathrm{m}$ for the $7.7 \mu \mathrm{m})$, the shape of the continuum and the integrated flux for each spectral band as taken from our images. The restrictions for the FWHM and the continuum are backed-up by a number of ISO observations in different environnements. We were not able to create a spectrum showing two emission bands on top of a continuum. This could be caused by an oversimplification of the problem, by a contamination of the LW5 filter by the $7.7 \mu \mathrm{m}$ band or by the real absence of the $6.2 \mu \mathrm{m}$ emission band. The second alternative is ruled out by the study of the relative distribution of the flux in the LW5 and LW6 filter: if the $7.7 \mu \mathrm{m}$ band was contaminating the LW5 filter, we should see a spatial correlation between the LW6 and the LW5 flux, which is not the case. The first alternative is probably ruled out also as we varied all the parameters of the fit without being able to reproduce a spectrum with two emission bands. Follow-up ISO observations are planned to put more constraints on the in-band intensities.

Whatever produces the emission in the three ISO filters, the intensity ratios we measure are not constant over the whole region (see fig 1b where we show the LW4/LW6 ratio). This implies that different excitation mechanisms contribute emission throughout the region, or that the dust properties vary spatially.

Finally, although the emission in the ISO filters resembles that in $\mathrm{H} \alpha$, the emission maximum in the $\mathrm{H} \alpha$ band is not cospatial with the maximum in the ISO filters. The maximum emission in the ISO filters is in fact at the location of the peak $\mathrm{CO}$ emission, therefore this indicates that either the $\mathrm{H} \alpha$ emission suffers substantial extinction, or the stars have mostly cleared out the HII region of dust.

\section{References}

Cohen, M., Bregman, J., Witteborn, F.C., Wooden, D.H., Rand, D.M., de Muizon, M.J., Allamandola, L. J., \& Tielens, A.G.G.M. 1989, Infrared Spectroscopy in Astronomy, pp. 149-154

Malumuth, E.M, Waller, W.H., \& Parker, J.W.M. 1996, AJ, 111, 1128

Metcalfe, L. et al. 1996, A\&A, 315, 105

Starck, J.L. et al., in preparation

Viallefond, F., Goss, W.M., van der Hulst, J.M., \& Crane, P.C. 1986, A\&AS, 64, 237

Wilson, C.D., \& Scoville, N. 1992, ApJ, 385, 512 